\title{
Design and Numerical Verification of Four States Polarization-independent Grating Coupler Using a Double-Layer Approach for a Single-photon Source based on HPW
}

Farooq Abdulghafoor Khaleel ( $\square$ farook.abd1101a@ilps.uobaghdad.edu.iq )

Institute of Laser for Postgraduate Studies https://orcid.org/0000-0003-3617-9439

Shelan Khasro Tawfeeq

Institute of Laser for Postgraduate Studies, University of Baghdad

\section{Research Article}

Keywords: Polarization-independent, grating coupler, photonic waveguide, integrated photonic, COMSOL, subwavelength structures

Posted Date: July 23rd, 2021

DOl: https://doi.org/10.21203/rs.3.rs-740232/v1

License: (c) (1) This work is licensed under a Creative Commons Attribution 4.0 International License.

Read Full License 


\title{
Design and numerical verification of four states polarization- independent grating coupler using a double-layer approach for a single-photon source based on HPW
}

\author{
Farooq Abdulghafoor Khaleel*, Shelan Khasro Tawfeeq** \\ Department of Engineering and Industrial Applications, Institute of Laser for \\ Postgraduate Studies, University of Baghdad, Baghdad, Iraq \\ *Farook.abd1101a@ilps.uobaghdad.edu.iq \\ * ORCID: 0000-0003-3617-9439 \\ ** ORCID: 0000-0003-0908-9518
}

Abstract: In this paper, a double-layer approach is proposed to design a compact four states polarization-independent grating coupler (GC). The proposed polarization-independent $\mathrm{GC}$ is designed to couple a $700 \mathrm{~nm}$ polarized light propagated in a $150 \mathrm{~nm}$ Gallium Phosphide $(\mathrm{GaP})$ waveguide to a polarizationmaintaining fiber (PMF). The double-layer approach is based on the deposition of $\mathrm{GaP}$ gratings designed to couple the transverse magnetic (TM) light over the $\mathrm{GaP}$ gratings designed to couple the transverse electric (TE) light. The two layers are separated by a Hydrogen silsesquioxane (HSQ) with an optimum thickness of 20 $\mathrm{nm}$. The proposed method resulted in relatively high coupling efficiencies (CEs) of $39.2 \%, 31.1 \%$, and $23.3 \%$ for the TE, TM, and $45^{\circ} / 45^{\circ}$ linearly polarized light, respectively. The polarization-dependent loss (PDL) is $1 \mathrm{~dB}, 1.26 \mathrm{~dB}$, and $2.26 \mathrm{~dB}$ corresponds to TE-TM, TM- $45^{\circ} / 45^{\circ}$, and $\mathrm{TE}-45^{\circ} /-45^{\circ}$, respectively. The performance of the double-layer approach is numerically verified by the twodimensional (2D) finite element algorithm (FEM) using COMSOL software. The proposed method suggests a novel and simple approach to design a compact four states polarization-independent GC that could be used in integrated (on-chip) photonic communication circuits. 
Keywords: Polarization-independent, grating coupler, photonic waveguide, integrated photonic, COMSOL, subwavelength structures

Declarations: The authors declare no conflict of interests. The simulation files generated during and/or analysed during the current study are available from the authors on reasonable request.

\section{Authors' contributions:}

Farooq A. Khaleel: Writing - original draft, Software, Validation. Shelan K. Tawfeeq: Data curation, Supervision, Conceptualization, Methodology, Writing review \& editing.

\section{Introduction:}

The optical fibers are widely used to transfer light over long distances with minimum propagation losses. The photonic waveguides perform the same function in on-chip photonic circuits. However, the coupling of the light between the optical fibers and the photonic circuits is a major issue because of the difference in their waveguide modes [1]. The GC is one of the best solutions that managed the coupling issue because of its high CE, compactness, easier alignment, and simple fabrication [2]. Moreover, a polarization-independent GC is needed in on-chip photonic circuits that its operations rely on the polarization state of the propagated light inside the photonic waveguide. For example, in [3], the emission of the quantum emitter (QE) embedded in the dielectric-loaded surface plasmon polariton (DLSPP) could be processed to produce a different polarization than the waveguide's output emission polarization.

The typical design of the GC supports the transmission of a single output polarization, only, because of the mismatch of the waveguide's effective index and, consequently, the grating period for each polarization [4-6]. However, several design strategies had been proposed to design a polarization-independent GC. For example, by tuning the fill factor and the grating period in $\mathrm{x}$ and $\mathrm{y}$ directions, a $64 \%$ 
polarization-independent $\mathrm{CE}$ could be achieved in the 2D GC proposed and simulated by 2D finite-difference time-domain (FDTD) in [7]. The tuning aimed to equalize the different effective indices for TE and TM transmitted light in a $340 \mathrm{~nm}$ $\mathrm{Si}$ waveguide for maximum $\mathrm{CE}$ at the operation wavelength.

Another approach to decrease the difference in the effective index for TE and TM polarization was simulated by FDTD in [8]. It was suggested and numerically proved that the increase of the Si channel thickness decreased the difference in the effective indices for TE and TM transmitted light and, consequently, a dualpolarization $\mathrm{CE}$ of $52.5 \%$ was achieved.

Moreover, a different complicated design strategy proposed in [9] resulted in a simulated $\mathrm{CE}$ of $60 \%$ for dual output polarization. This approach suggested corrugating the top and bottom surfaces of the $370 \mathrm{~nm}$ Si waveguide embedded in silicon oxide $\left(\mathrm{SiO}_{2}\right)$ to form the 1D TE and TM grating periods. The tuning of the etch depths of the TE and TM gratings in the top and bottom of the Si channel resulted in a common maximum $\mathrm{CE}$ for both polarization cases. Despite the outstanding design and numerical performance, the sophisticated photonic structures found difficulties in the experimental fabrication.

Another design strategy [10] achieved a simulated CE of $20 \%$ for TE and TM light polarization. This strategy was based on the geometrical union or intersection for the designed TE and TM 1D grating periods in a $220 \mathrm{~nm} \mathrm{Si}$ waveguide. Although this outstanding approach solved the grating's polarization dependency, however, it results in a nonuniform gratings (apodized) periodicity with thin grooves or summits in the Si waveguide. Consequently, the apodization of the GCs adds to the complexity of the fabrication when it is desired to use this approach in other different wavelengths since smaller operating wavelengths result in thinner grating grooves or summits [11]. 
A recent study on a $400 \mathrm{~nm}$ thick silicon waveguide channel aimed to design a subwavelength 1D polarization-independent GC [12]. This approach was based on the equalization of the effective refractive indices for TE and TM modes and a simulated CE of $50 \%$ for dual output polarization was obtained. The same approach was performed on a $460 \mathrm{~nm}$ thick silicon waveguide and a simulated CE of $60 \%$ was achieved [13].

A recent approach utilized a $220 \mathrm{~nm} \mathrm{Si}$ waveguide channel to design a dualpolarization 1D GC [14]. This approach deposited secondary subwavelength gratings in the lower index region of the primary grating, where the high index region of the primary grating was filled with Si completely. By optimizing the filling factors and grating periods for both the primary and secondary gratings, a maximum CE of $32.5 \%$ was simulated for both TE and TM modes.

Finally, a 2D subwavelength GC based on a $220 \mathrm{~nm} \mathrm{Si} \mathrm{waveguide} \mathrm{was}$ recently proposed in [15]. This new approach modified the etch depths, grating periods, and fill factors of the gratings in 2D to achieve a shared effective index of the grating for TE and TM modes and in dual operation wavelengths of $1330 \mathrm{~nm}$ and $1550 \mathrm{~nm}$. This approach simulated a maximum CE of $35.5 \%$ for both polarization cases and efficiently acted as a wavelength demultiplexer.

It is worth mentioning that all the aforementioned highly informative publications operated at C-band or O-band wavelengths. Recently emerged nanophotonic applications urged the need for polarization-independent GCs at different wavelengths. For example, the single-photon emitter embedded in a DLSPP or hybrid plasmonic waveguide [16] requires a polarization-insensitive GC to support the emission of photons with different output polarization. This is important in designing on-chip quantum key distribution systems based on polarization-dependent protocols such as the BB84 protocol. The on-chip BB84 
protocol requires the GC to support the emission of four states of polarization, which are $0^{\circ}, 45^{\circ},-45^{\circ}$, and $90^{\circ}[17,18]$.

In this paper, a novel, and simple approach is proposed to design a compact four states polarization-independent GC based on a double-layer approach utilizing a $150 \mathrm{~nm} \mathrm{GaP}$ waveguide. The deposition of the second GaP layer designed to couple a TM polarized light above the first GaP layer designed to couple a TE polarized light emits the light efficiently regardless of its polarization state. Also, this approach yields uniform grating periods instead of thin or apodized grating structures. Consequently, the fabrication process is easier with multiple depositions and etching processes. To the best of our knowledge, this is the first approach that investigated the design of a polarization-independent GC at the visible wavelength with four states of output polarization. This paper is arranged as follows: Section (2) describes the theoretical design principle of the double-layer approach. Section (3) presents the 2D COMSOL framework settings, simulation results and discusses the findings. Finally, Section (4) concludes the paper.

\section{Theoretical design methodology:}

GCs are formed by periodic alteration in the refractive index or the geometrical configuration of the waveguide's channel. The incident wave's phase is diffracted to several spatial directions because of that alteration. The Bragg condition specifies diffracted waves number as [19]:

$$
\mathrm{k}_{\mathrm{diff}}=\mathrm{k}_{\mathrm{inc}}+\mathrm{nk}_{\mathrm{g}}
$$

Where $\mathrm{k}_{\mathrm{diff}}$ is the diffracted wave's wavevector, $\mathrm{k}_{\mathrm{inc}}$ is the incident wave's wavevector, $\mathrm{k}_{\mathrm{g}}$ is the grating's wavevector and equals $2 \pi / \Lambda$, where $\Lambda$ is the grating's period and $\mathrm{n}$ is the diffraction order, i.e., $\mathrm{n}=0, \pm 1, \pm 2, \ldots, \infty$. Although the grating coupler is a $3 \mathrm{D}$ structure, however, the following work is based on a $2 \mathrm{D}$ simulation 
framework since it was assumed that the GC's third dimension is larger than the operating wavelength by many orders [20].

Furthermore, it is assumed that the GC is connected to the photonic waveguide by an ideal taper section. Because of the reciprocity principle, the GC could be used to diffract the light propagating in the photonic waveguide towards the optical fiber and vice versa [21]. The proposed GC based on the double-layer approach is shown in Figure (1).

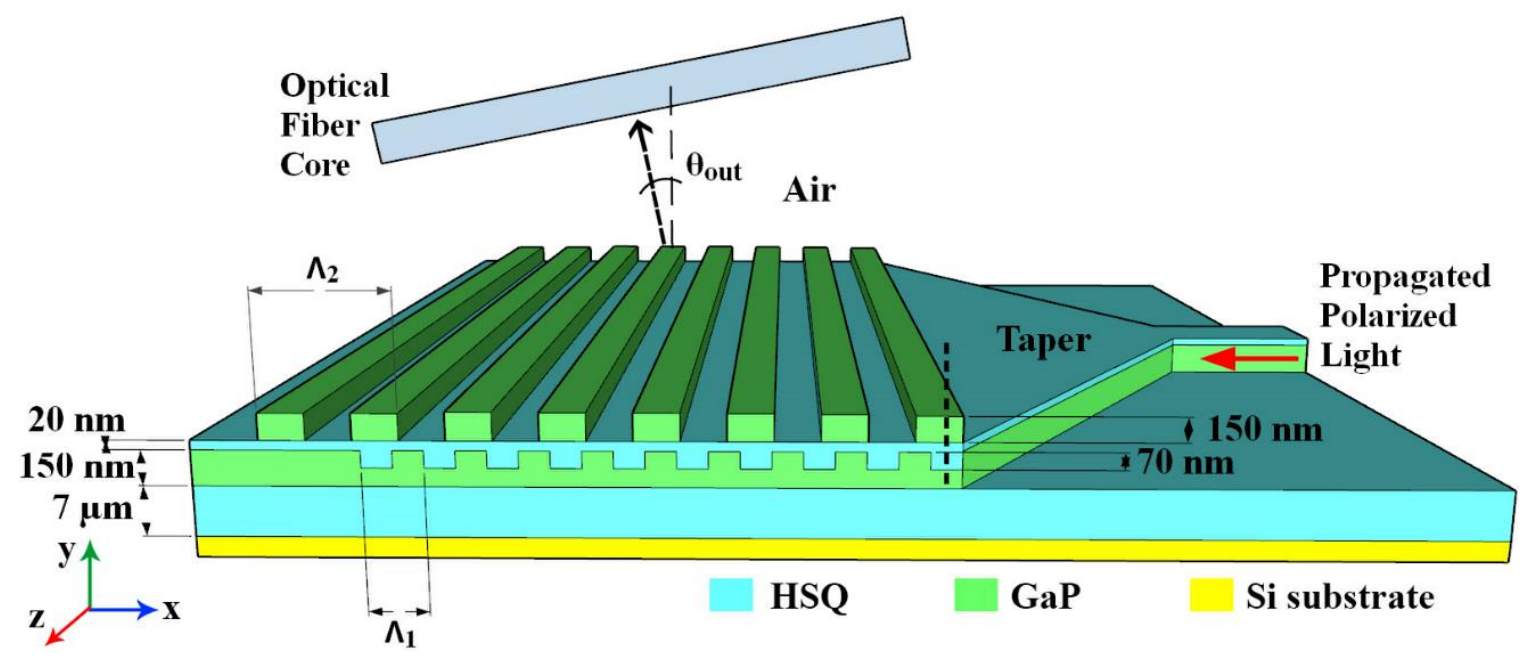

Figure 1: The proposed double-layer GC

The goal is to maximize the collection efficiency of the diffracted polarized light by a PMF. The PMF is tilted by the angle $\left(\theta_{\text {out }}=10^{\circ}\right)$ to avoid higher diffraction orders from the GC. Furthermore, the PMF modeling data are based on the PM630HP Thorlabs fiber datasheet. The PM630-HP fiber has a mode field diameter (MFD) of $4.5 \mu \mathrm{m}$, a core diameter of $3.5 \mu \mathrm{m}$, and operation wavelengths from $620 \mathrm{~nm}$ to $850 \mathrm{~nm}$ [22]. Moreover, a $1 \mu \mathrm{m}$ normal distance from the GC is considered for the PMF. The following simulations are based on an operation wavelength $(\lambda)$ of 700 $\mathrm{nm}$. This $\lambda$ is widely used as a general emission $\lambda$ for the QEs embedded inside a DLSPP or HPW waveguides $[3,16]$. Besides, an HSQ layer with an optimum thickness of $20 \mathrm{~nm}$ is deposited above the first GC layer to act as a separator between 
the two GCs layers [23]. The refractive indices at $700 \mathrm{~nm}$ for both GaP and HSQ are 3.2543 and 1.41, respectively. A user-defined port at the right side injects the light into the GaP photonic waveguide with different polarization configurations. The user-defined port mimics the QE's emission with different polarization propagated in the HPWs investigated in [23].

Before depositing the second GC layer composed of $150 \mathrm{~nm} \mathrm{GaP}$ gratings, the first GC layer should be designed to support the coupling of a TE polarized light. The first GC layer's period $\left(\Lambda_{1}\right)$ could be calculated from [20]:

$$
\Lambda_{1}=\frac{\lambda}{n_{(\text {eff-TE })}-n_{\text {HSQ }} \sin \left(\theta_{\text {out }}\right)}
$$

Where $\mathrm{n}_{(\text {eff-TE) }}$ is the effective mode index for the TE mode, and $\mathrm{n}_{\mathrm{HSQ}}$ is the refractive index of the HSQ layer. The $\mathrm{n}_{(\mathrm{eff}-\mathrm{TE})}$ is calculated from [20]:

$$
\mathrm{n}_{(\text {eff-TE })}=\mathrm{ff}_{\text {high }} \mathrm{n}_{(\text {eff-full channel })}+\left(1-\mathrm{ff}_{\text {high }}\right) \mathrm{n}_{(\text {etched channel })}
$$

Where $\mathrm{n}_{(\text {eff-full channel) }}$ is the effective index of the $150 \mathrm{~nm} \mathrm{GaP}$ region, $\mathrm{ff}_{\text {high }}$ is the fill factor of the $150 \mathrm{~nm} \mathrm{GaP}$ region, and $\mathrm{n}_{\text {(etched channel) }}$ is the effective index of the $80 \mathrm{~nm} \mathrm{GaP}$ channel. The $70 \mathrm{~nm}$ edge depth is the optimized etch depth that corresponds to the highest $\mathrm{CE}$ as will be explained in the following simulations. The effective indices for each structure are calculated by the slab waveguide approximation [1] using the 2D mode analysis tool in COMSOL as shown in Figure (2). 
Air $\square$ GaP $\square$ HSQ
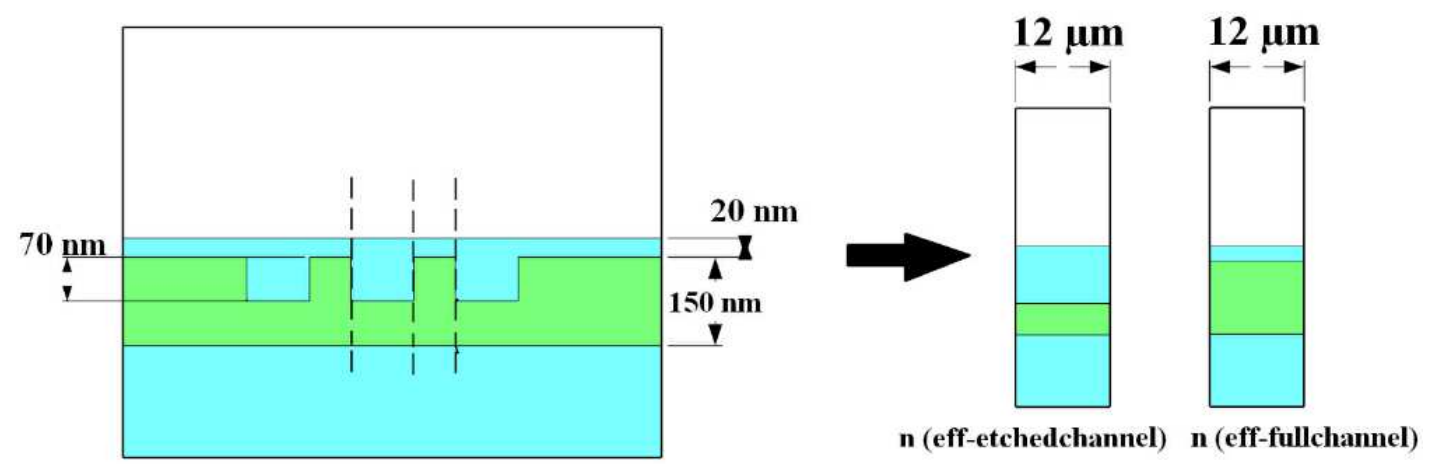

Figure 2: The separation of the GC into two slab waveguides to estimate the effective index for the full channel and etched channel waveguides

The effective index for the TM polarization is smaller than the effective index in the TE polarization. A smaller TM effective index results in a larger grating period to efficiently couple the TM polarized light to the PMF. However, it is possible to equalize the effective indices for both TE and TM polarization cases by depositing a second layer composed of $150 \mathrm{~nm} \mathrm{GaP}$ gratings above the $20 \mathrm{~nm}$ HSQ separator. The deposition of the second layer results in a modification in the effective index in Equation (2) that makes the GC compatible to transmit the TE or the TM light with a high $\mathrm{CE}$. The addition of the second GaP layer modifies the calculation of the effective mode index. The new GC structure should be divided into four regions as shown in Figure (3) to calculate the overall effective index in Equation (2).

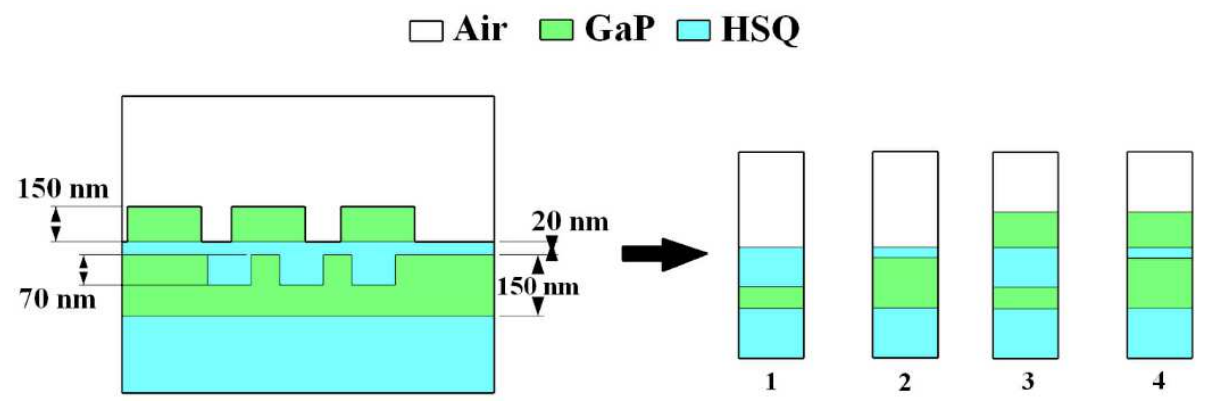

Figure 3: The new separation of the GC into four slab waveguides

Consequently, Equation (3) is modified as: 


$$
\begin{gathered}
\mathrm{n}_{(\text {eff })}=\mathrm{ff}_{1} \mathrm{n}_{(\text {eff-1) }}+\mathrm{ff}_{2} \mathrm{n}_{(\text {eff-2) }}+\mathrm{ff}_{3} \mathrm{n}_{(\text {eff-3) }}+\mathrm{ff}_{4} \mathrm{n}_{(\text {eff-4) }}+ \\
\cdots+\mathrm{ff}_{\mathrm{n}} \mathrm{n}_{(\text {eff-n })} \\
\mathrm{ff}_{1}+\mathrm{ff}_{2}+\mathrm{ff}_{3}+\mathrm{ff}_{4}+\cdots+\mathrm{ff}_{\mathrm{n}}=1
\end{gathered}
$$

Where $\mathrm{n}_{(\mathrm{eff})}$ is the total effective index of the new $\mathrm{GC}, \mathrm{ff}_{1,2,3,4}$ are the fill factors of the regions from 1 to 4 , respectively, and $n_{(\text {eff-1,2,3,4) }}$ are the effective indices for each region shown in Figure (3), respectively. Moreover, the resultant new structure is not periodic, and Equations (4.a) and (4.b) should include all the resultant random fill factors over all the GC's corrugations.

With the proposed modification, it is possible to tune the grating periods and the fill factors for the double layers to achieve the optimum values for maximum $\mathrm{CE}$ for four polarization cases. The described approach simplifies the fabrication process since it yields a uniform GC for both layers. Non-uniform or apodized GCs approaches might face difficulties in fabrication processes or optimizations since their geometries are based on thin waveguide grooves or summits that could not be fabricated with current technologies [21].

\section{Simulation results and discussion:}

The 2D COMSOL framework is used to verify the results of the proposed GC shown in Figure (1). The simulation region boundaries are set to the second-order scattering boundary conditions to scatter the diffracted waves in space without any back reflections to the GC's structure. Besides, the mesh settings are set as a Physicscontrolled mesh with an extremely fine resolution for accurate calculation of the propagated and scattered fields. Numeric ports are used to excite the TE, TM, and a $45^{\circ} /-45^{\circ}$ linearly polarized light in the GaP waveguide channel. The diagram presented in Figure (4) describes the proposed design strategy. 


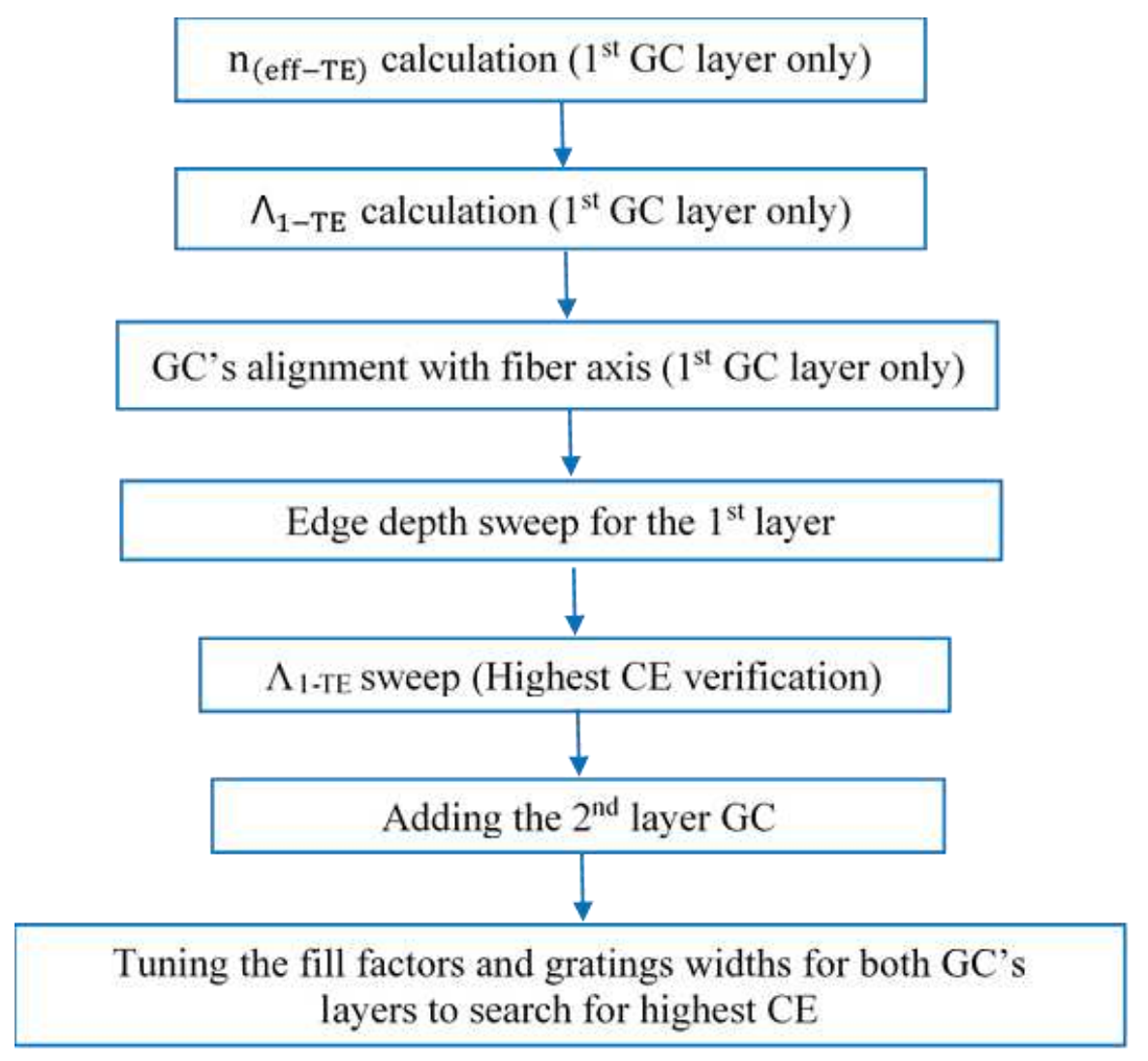

Figure 4: The proposed double-layer GC's design strategy

The initial etch depth is selected to be half the channel width of the $150 \mathrm{~nm} \mathrm{GaP}$ photonic waveguide described in [23]. Firstly, the effective index calculation is based on the slab waveguide approximation. The resultant indices for the $150 \mathrm{~nm}$ and $75 \mathrm{~nm} \mathrm{GaP}$ channel waveguides for the TE mode are shown in Figure (5).

(a)

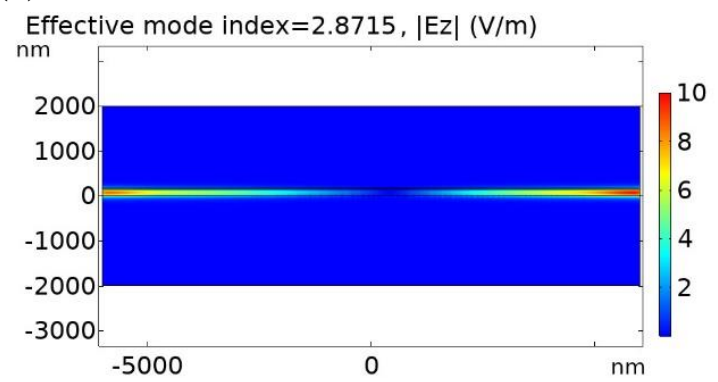

(c) (b)

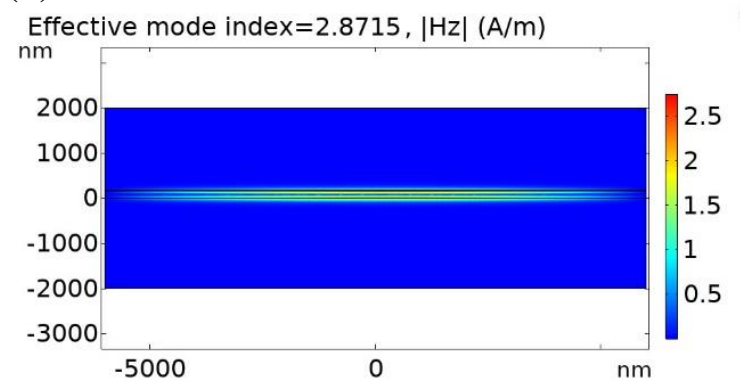

(d) 

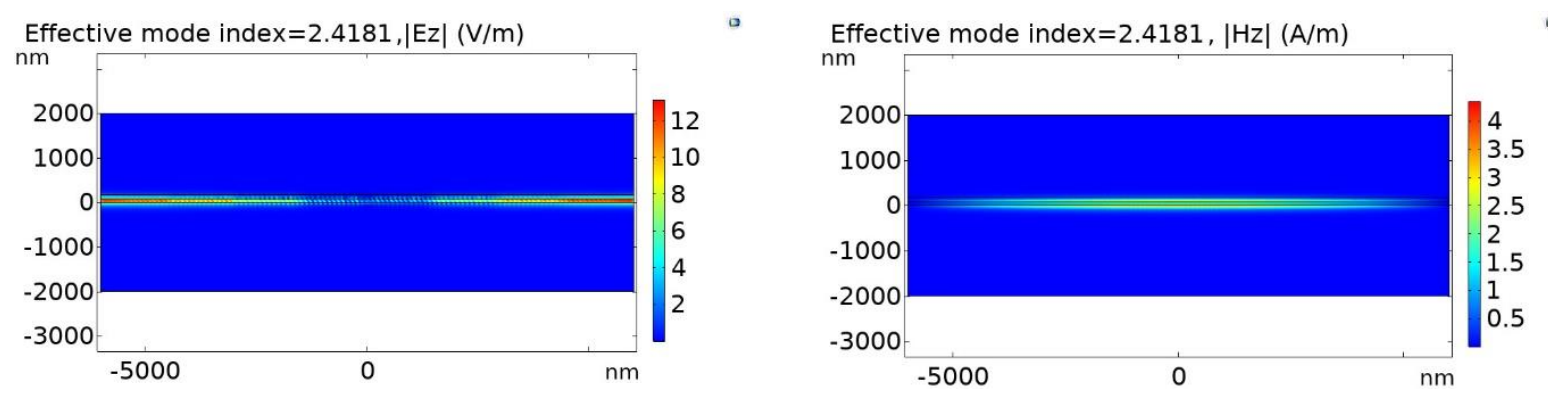

Figure 5: The z-component of the electric and magnetic field for (a) and (b) unetched GaP waveguide (150 nm), (c), and (d) etched GaP waveguide (75 $\mathrm{nm})$, respectively, and their corresponding effective index

The resultant effective index is determined from Equation (3) is 2.6448. Consequently, the GC period that supports the TE polarization is $283.3 \mathrm{~nm}$ for a fill factor of 0.5 as Equation (2) implies. The initial $\Lambda_{1}$ is selected as $280 \mathrm{~nm}$ because the short and sub-nm lengths could occur due to fabrication error.

The alignment of the GC to the fiber in the simulation domain is necessary to find the optimum spatial x-position coordinate (the dotted line in Figure (1)) to achieve a maximum CE. Consequently, Figure (6) shows the $\mathrm{CE}$ of the GC against the $\mathrm{x}$-axis spatial coordinate of the GC to a fixed $\mathrm{x}$-coordinate of the PMF. The $\mathrm{x}$ axis spatial coordinate is varied from $920 \mathrm{~nm}$ to $1060 \mathrm{~nm}$ which corresponds to higher CE for a TE mode. 


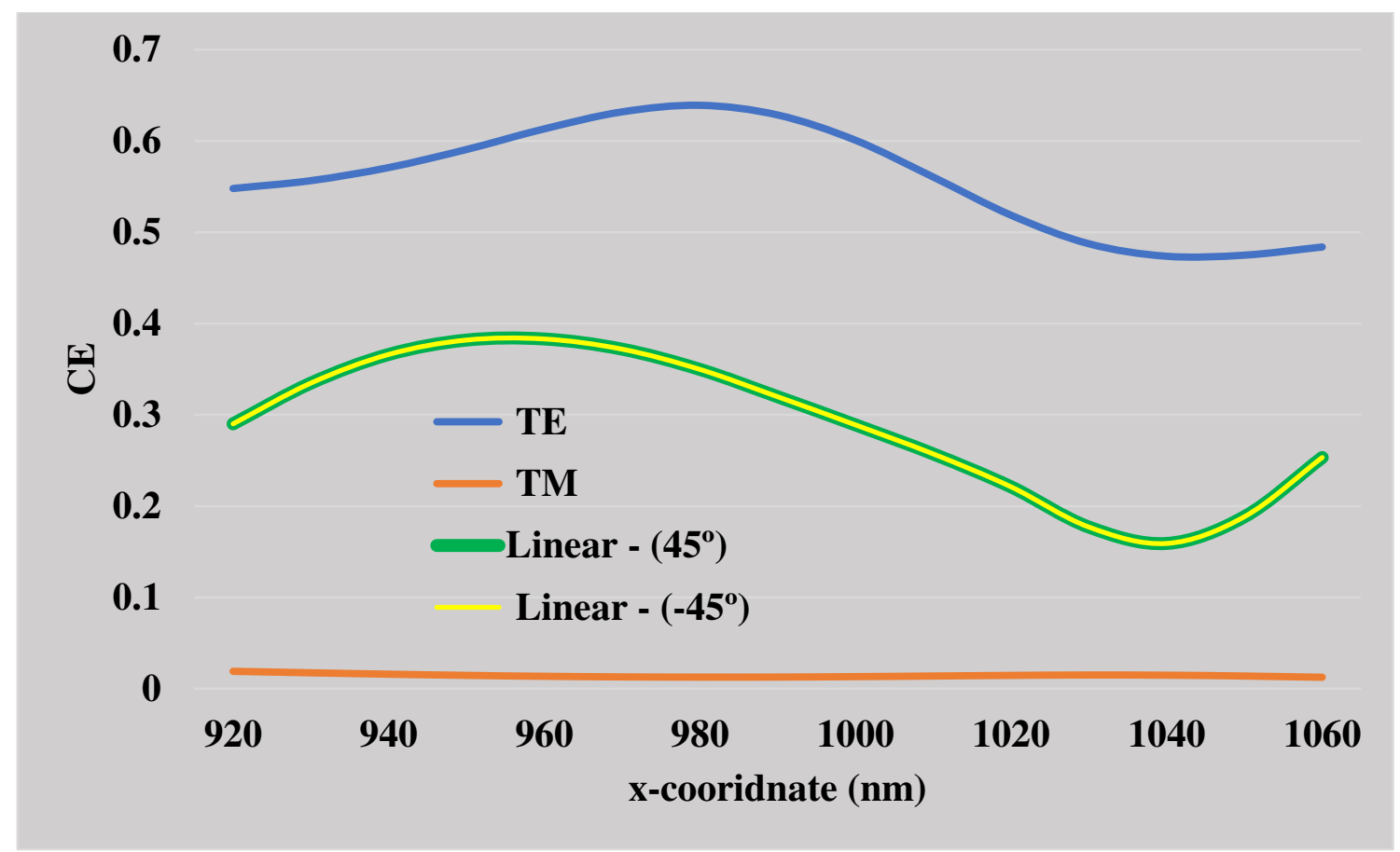

Figure 6: The CE of a GC against the x-coordinate to a fixed position of a PMF in the simulation domain

The highest CE is at $980 \mathrm{~nm}$, which is fixed in the following simulations. The number of gratings to the left side from the optimum x-position is selected such that the GC with a minimum $\Lambda$ covers the entire core of the PMF. Consequently, 11 gratings of $280 \mathrm{~nm}$ for the $1^{\text {st }}$ layer and a similar number of gratings for the $2^{\text {nd }}$ layer. Also, 11 gratings are enough to diffract most of the propagated light inside the $\mathrm{GaP}$ waveguide.

The etch depth is swept from $30 \mathrm{~nm}$ to $90 \mathrm{~nm}$ with a $5 \mathrm{~nm}$ step size for maximum $\mathrm{CE}$ as shown in Figure (7). 


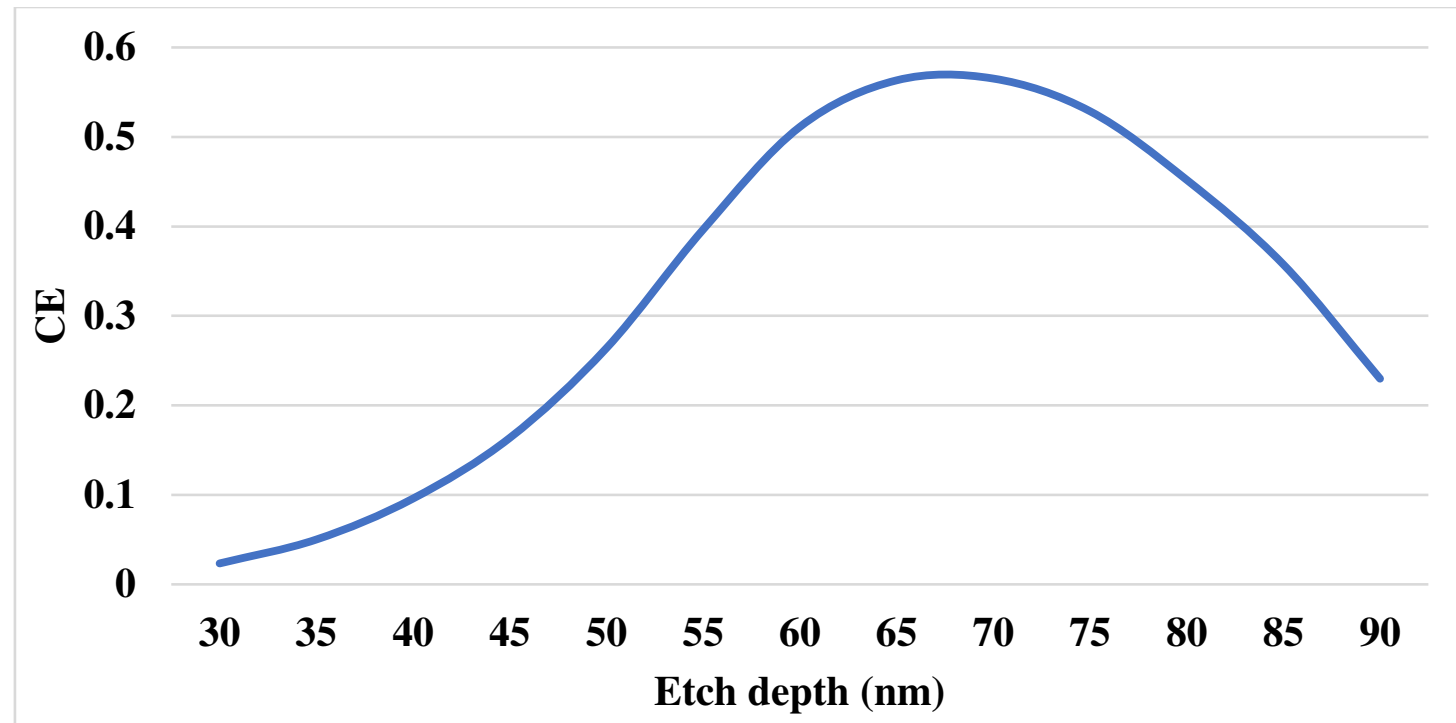

Figure 7: The CE against the etch depth of the first layer only

Figure (7) shows a maximum CE of 56.52\% for an etch depth of $70 \mathrm{~nm}$. By setting the etch depth and the x-position to their optimal values, the GC period is swept from $275 \mathrm{~nm}$ to $300 \mathrm{~nm}$ with a $5 \mathrm{~nm}$ step size to search for maximum CE as shown in Figure (8).

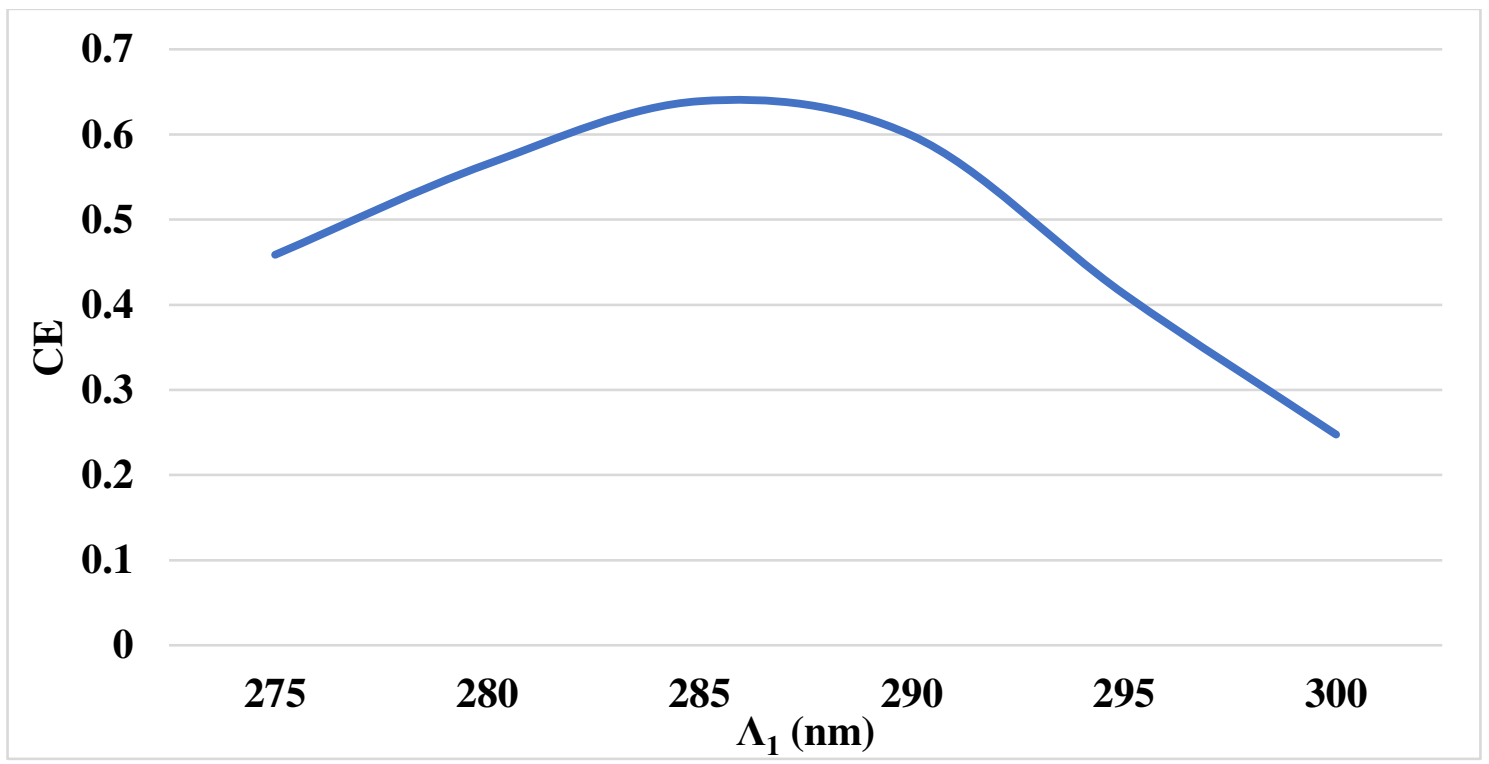

Figure 8: The CE against $\Lambda_{1}$ 
Figure (8) shows a maximum CE of $64 \%$ for a $\Lambda_{1}$ equals $285 \mathrm{~nm}$. By setting all the optimal parameters of $\Lambda_{1}$, etch depth, and the GC's x spatial position of the first layer only, the CE for a TE-polarized light against the operation wavelength is shown in Figure (9) with a maximum CE of $64 \%$ at $700 \mathrm{~nm}$.

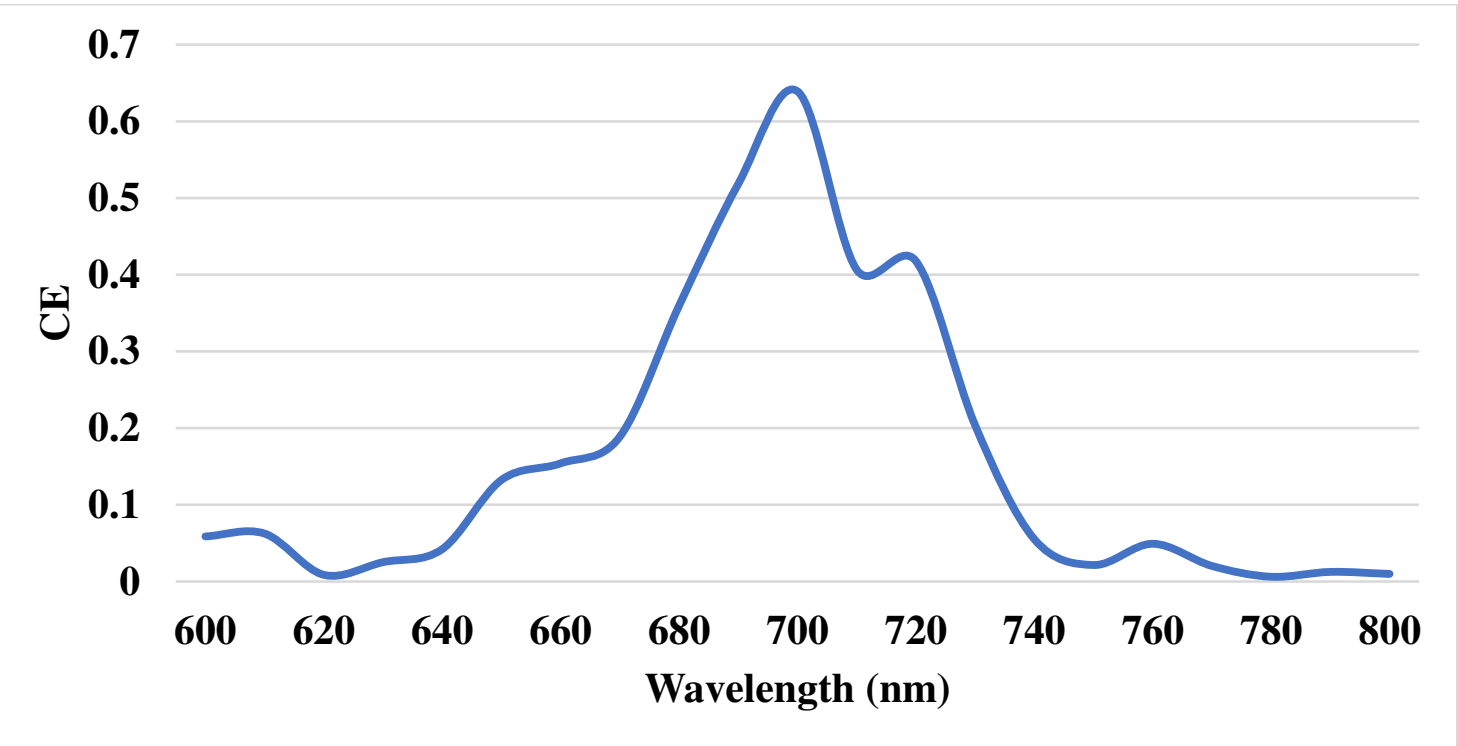

Figure 9: The CE against the operation wavelength for a TE polarization excitation from the right port

The second layer should be designed to support the coupling of a TM polarized light. The corresponding $\Lambda_{2}$ should be changed because $n_{\text {eff }}$ for the TM mode differs from that of the TE mode. The effective indices for the unetched (150 $\mathrm{nm})$ and etched $(150-70=80 \mathrm{~nm}) \mathrm{GaP}$ waveguide for a TM mode are shown in Figure (10).

(a)

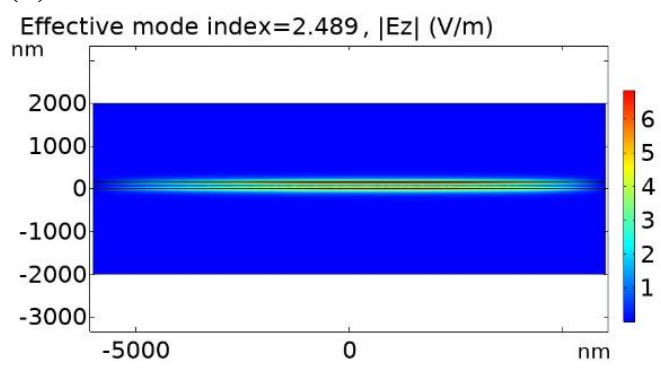

(c) (b)

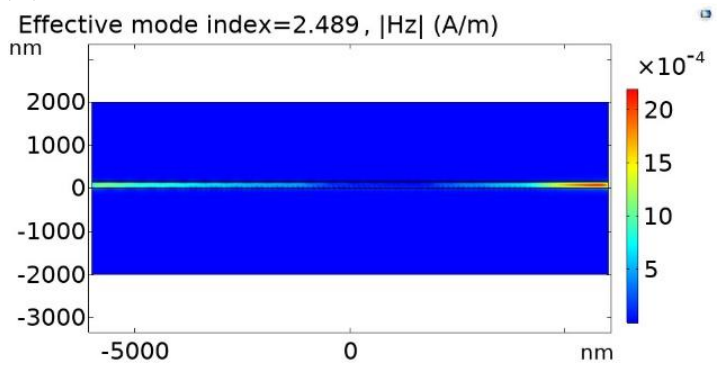

(d) 

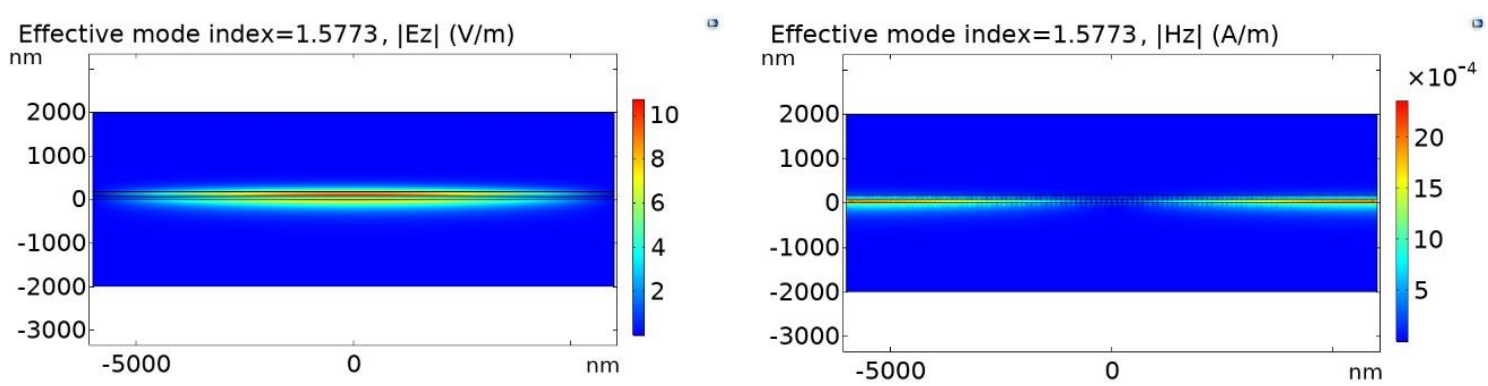

Figure 10: The z-component of the electric and magnetic field for (a) and (b) unetched $\mathrm{GaP}$ waveguide, (c) and (d) etched $(80 \mathrm{~nm}) \mathrm{GaP}$ waveguide, respectively, and their corresponding effective index

For an $\mathrm{ff}$ of $50 \%$, the $\Lambda_{2}$ is obtained from Equations (4.5) and (4.8), which equals $376.45 \mathrm{~nm}$. The following simulations select $\Lambda_{2}$ to be $375 \mathrm{~nm}$.

Figure (11) shows the CE spectra for each case of excitation polarization using the single-layer approach and the double-layer approach.

(a)

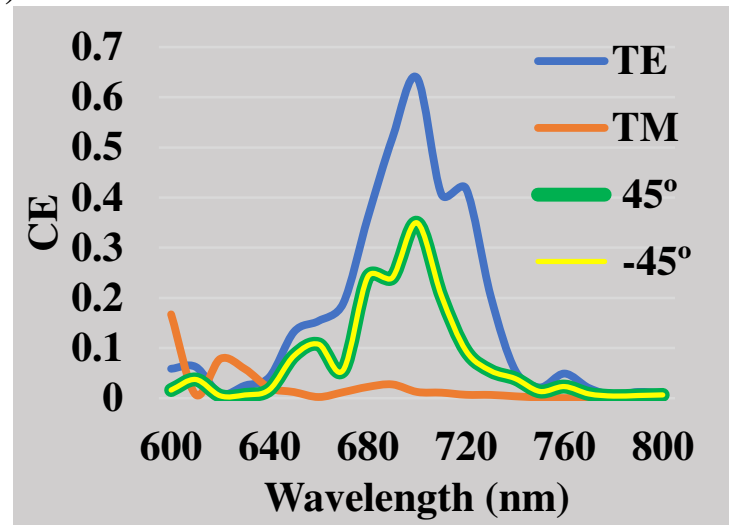

(b)

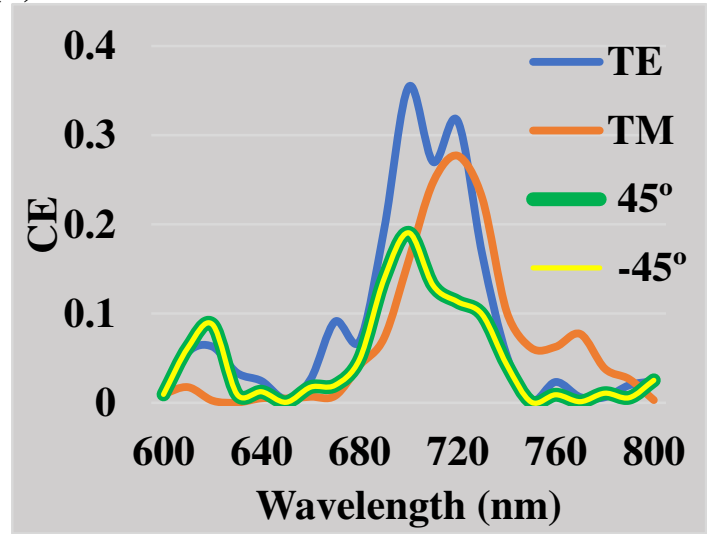

Figure 11: The CE spectra of the proposed GC with (a) a single-layer approach, (b) a doublelayer approach

The single-layer approach of the GC achieved a CE of $64 \%$ for a TE polarized light, $35 \%$ for a linearly polarized light at $45^{\circ} /-45^{\circ}$, and $1 \%$ for a linearly polarized light at $90^{\circ}$ as shown in Figure (11.a).

A higher CE for TM polarized light could be achieved by depositing a second layer of $150 \mathrm{~nm} \mathrm{GaP}$ with a grating period of $375 \mathrm{~nm}$ above the first layer with the same thickness as shown in Figure (1). However, the CEs for TE and $45^{\circ} /-45^{\circ}$ 
linearly polarized light deteriorate. CEs of $35 \%, 16 \%$, and $19 \%$ for the TE, TM, and $45^{\circ} /-45^{\circ}$ linearly polarized light, respectively, are achieved as shown in Figure (11.b).

By tuning the filling factors, $\Lambda_{1}$, and $\Lambda_{2}$, better performance for the proposed GC could be achieved. The fill factor for each layer is tuned from 0.1 to 0.9 with a 0.1 step size, while the grating periods of the first layer and the second layer are tuned from $260 \mathrm{~nm}$ to $310 \mathrm{~nm}$ and from $350 \mathrm{~nm}$ to $400 \mathrm{~nm}$, respectively. The corresponding CEs for each polarization case are recorded in separate EXCEL sheets for each case. For example, for an ff of 0.5 for the first layer, an ff of 0.6 for the second layer, and the grating period for the first and second layer are tuned to 285 $\mathrm{nm}$ and $380 \mathrm{~nm}$, respectively, the CEs are $39.2 \%, 31 \%$ and $23.3 \%$ for a TE, TM and $45^{\circ} /-45^{\circ}$ linearly polarized light, respectively, as shown in Figure (12).

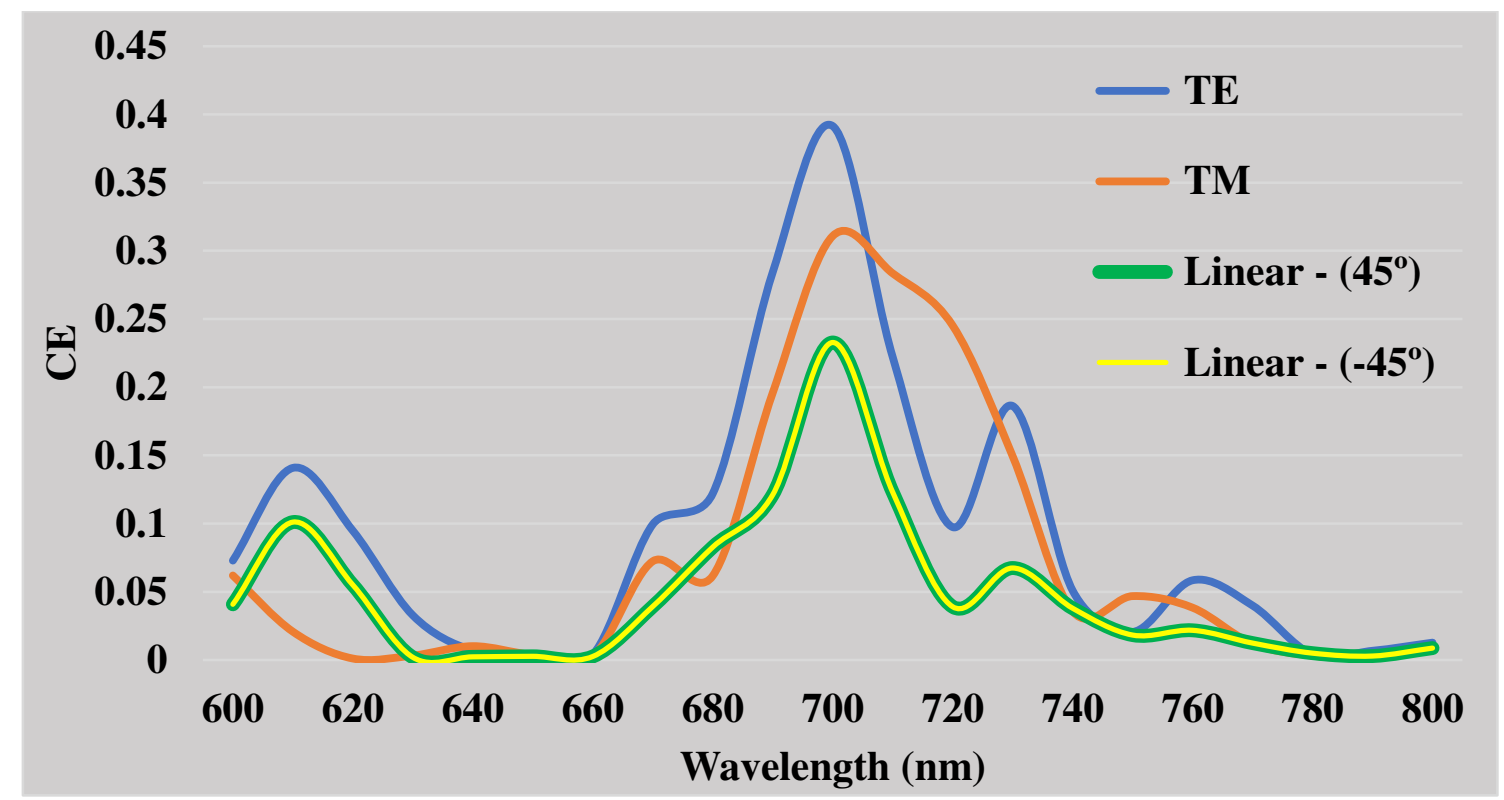

Figure 12: The CE spectra of the four polarization cases for the optimum double-layer GC

The polarization-dependent loss (PDL) identifies the polarizationindependence of the GC and is defined as $[12,13]$ :

$$
\mathrm{PDL}=10 \log \left|\frac{\mathrm{CE}_{\mathrm{TE}}}{\mathrm{CE}_{\mathrm{TM}}}\right|
$$




$$
\begin{aligned}
& \mathrm{PDL}=10 \log \left|\frac{\mathrm{CE}_{\mathrm{TM}}}{\mathrm{CE}_{45^{\circ} /-45^{\circ}}}\right| \\
& \mathrm{PDL}=10 \log \left|\frac{\mathrm{CE}_{\mathrm{TE}}}{\mathrm{CE}_{45^{\circ} /-45^{\circ}}}\right|
\end{aligned}
$$

Accordingly, the optimum double-layer GC shows a PDL of $1 \mathrm{~dB}, 1.25 \mathrm{~dB}$, and 2.26 $\mathrm{dB}$ for TE-TM, TM- $45^{\circ} /-45^{\circ}$, and TE- $45^{\circ} /-45^{\circ}$, respectively, at $700 \mathrm{~nm}$ as shown in Figure (13).

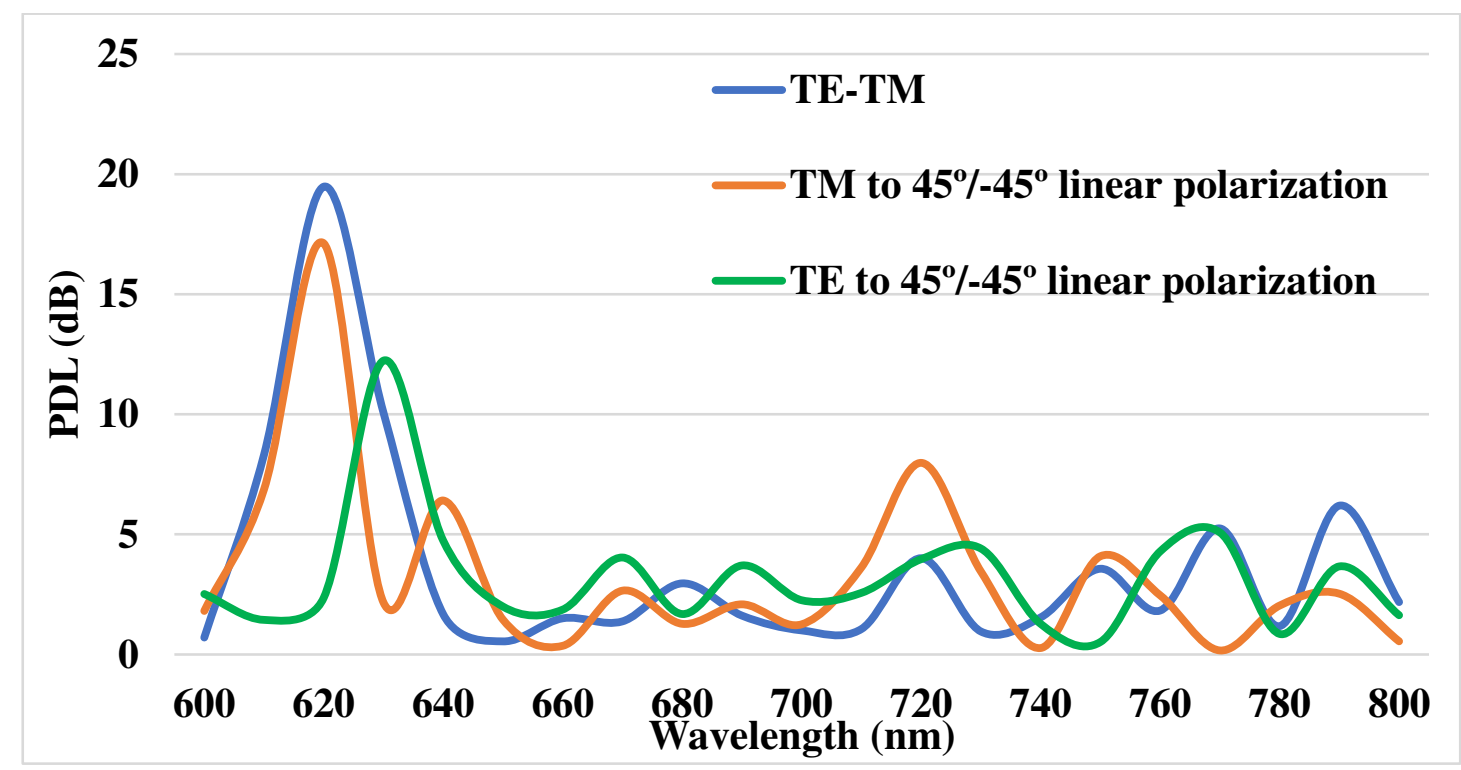

Figure 13: The PDL performance of the optimum double-layer GC for different polarization cases

The observation of the intensity in the simulation domain is necessary to show how the fields are scattered. Figure (14) shows the intensity distribution of the light for each case of polarization of the excitation source (Right port).

(a)

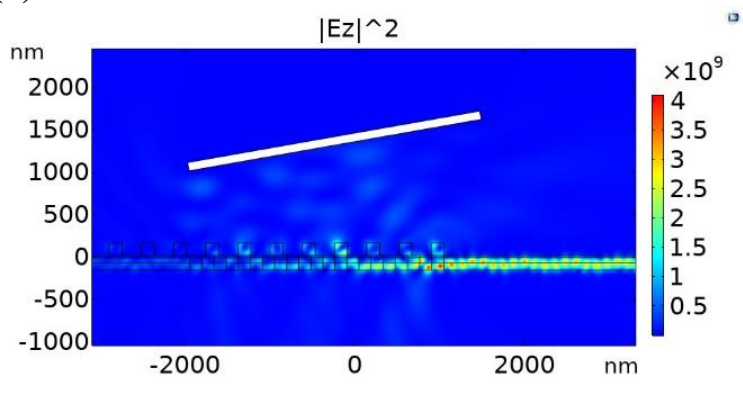

(b)

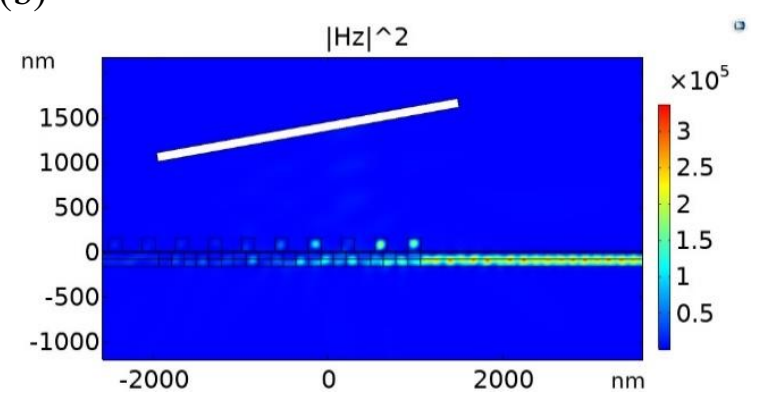


(c)

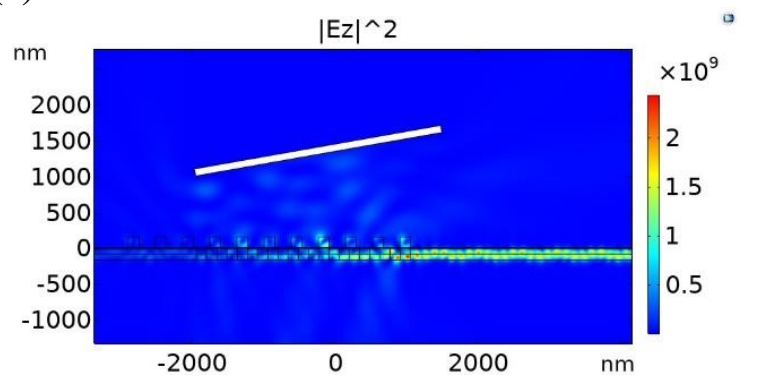

(d)

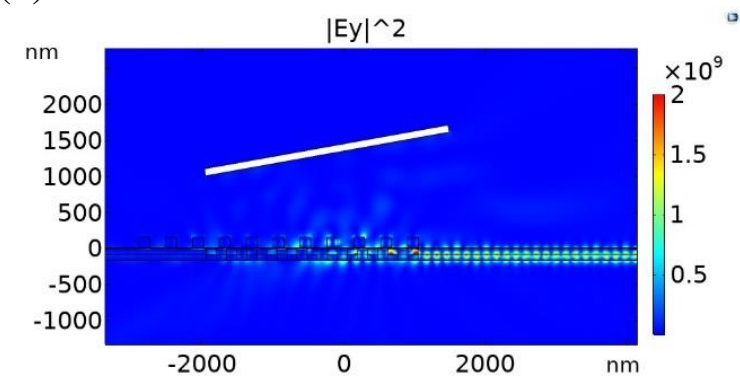

Figure 14: The intensity distribution in the simulation domain for (a) a TE excitation, $(b) a$ TM excitation, (c), and (d) a $45^{\circ}$ linear excitation

Figure (14.a) shows the scattering of the $\left|\mathrm{E}_{\mathrm{z}}\right|^{2}$ in the case of $0^{\circ}$ polarization excitation from the right port. The out-of-plane component of the field, which is $\mathrm{E}_{\mathrm{z}}$, generates both $\mathrm{H}_{\mathrm{x}}$ and $\mathrm{H}_{\mathrm{y}}$ components inside the GaP region. However, the $\mathrm{H}_{\mathrm{y}}$ component is the major field in the selected mode of propagation. Consequently, the $\mathrm{H}_{\mathrm{y}}$ component domination is a clear indication that $39 \%$ of the propagated photons polarized at $0^{\circ}$ inside the GaP region are coupled to the PMF. Similarly, Figure (14.b) shows the scattering of the $\left|\mathrm{H}_{\mathrm{z}}\right|^{2}$ in the case of $90^{\circ}$ excitation. For a $45^{\circ}$ linearly polarized light, the excitation of the right port is performed with both $\mathrm{E}_{\mathrm{y}}$ and $\mathrm{E}_{\mathrm{z}}$ components of the light are activated.

Accordingly, Figures (14.c) and (14.d) show the scattering of the z-component and $y$-component of the intensity, respectively. Moreover, for a $-45^{\circ}$ linearly polarized light, the excitation of the right port is similar to that in the $45^{\circ}$ polarization case except that the $\mathrm{z}$-component of the excitation source becomes negative. As a result, the scattering of the field in the simulation domain is similar to that shown in Figures (14.c) and (14.d) except for the negativity of the $E_{z}$ component to Figure (14.c). Consequently, the intensity distribution for the $-45^{\circ}$ polarization case was not included in Figure (14) to concise. Moreover, there is a negligible scattering of the intensity field in the space of the simulation domain (air region) for all the polarization cases. 
Table (4.1) compares the performance of the proposed double-layer GC approach with other polarization-independent GCs approaches. To the best of our knowledge, the proposed work investigated the polarization-independence issue with four output polarization states and at a different emission wavelength rather than the GCs designed to couple the TE/TM polarization only at C-band or O-band wavelengths.

Table 1: A summary for the simulation performance of the polarization-independent GCs approaches

\begin{tabular}{|c|c|c|c|}
\hline Publication & Wavelength $(\mathrm{nm})$ & Waveguide channel width (nm) & CE (\%) \\
\hline$[7]$ & 1550 & 340 & 64 \\
\hline$[8]$ & 1550 & 750 & 52.5 \\
\hline$[9]$ & 1550 & 370 & 60 \\
\hline$[10]$ & 1550 & 220 & 20 \\
\hline$[12]$ & 1550 & 400 & 60 \\
\hline$[13]$ & 1550 & 460 & 60 \\
\hline$[14]$ & 1550 & 220 & 32.5 \\
\hline$[15]$ & 1550 & 220 & 35.5 \\
\hline Our work & 700 & 150 & $39.2 * / 31^{* * / 23.3 * * *}$ \\
\hline
\end{tabular}

* For a TE polarization

** For a TM polarization

$* * *$ For a $45^{\circ} /-45^{\circ}$ linear polarization

\section{Conclusion:}

A novel and, a simple approach is proposed to design a compact and polarization-independent GC. The proposed method does not yield geometries with ultrathin edges in the waveguide section. Consequently, the proposed method simplifies the fabrication process through consecutive deposition and etching processes. Furthermore, the proposed design methodology could be followed as a general design methodology for any emission wavelength or waveguide dimensions. The CE of the proposed GC is comparable to other GCs presented in the literature survey with larger channel width. Different approaches could be followed to improve the performance of the proposed GC. For example, the second layer 
gratings' shape could be altered to obtain equal CEs and PDL for the four polarization cases. Consequently, the new shape might result in equal brightness for all polarization cases.

\section{References:}

[1] L. Chrostowski, M. Hochberg, Silicon Photonics Design: From Devices to Systems, Cambridge University Press, 2015. https://doi.org/10.1017/CBO9781316084168.

[2] G. Roelkens, D. Van Thourhout, Interfacing Silicon Nanophotonic Integrated Circuits and Single-Mode Optical Fibers with Diffraction Gratings BT Silicon Photonics II: Components and Integration, in: D.J. Lockwood, L. Pavesi (Eds.), Springer Berlin Heidelberg, Berlin, Heidelberg, 2011: pp. 7194. https://doi.org/10.1007/978-3-642-10506-7_3.

[3] H. Siampour, O. Wang, V.A. Zenin, S. Boroviks, P. Siyushev, Y. Yang, V.A. Davydov, L.F. Kulikova, V.N. Agafonov, Ultrabright single-photon emission from germanium-vacancy zero-phonon lines : deterministic emitterwaveguide interfacing at plasmonic hot spots, Nanophotonics. (2020) 1-10. https://doi.org/10.1515/nanoph-2020-0036.

[4] J. Xu, X. Jin, Y. Zhao, Apodized fully-etched surface grating coupler using subwavelength structure for standard silicon-on-insulator waveguide, Opt. Quantum Electron. 49 (2017) 158. https://doi.org/10.1007/s11082-017-09974.

[5] Y. Xu, F. Wang, Y. Gao, W. Chen, C. Chen, X. Wang, Y. Yi, X. Sun, D. Zhang, Efficient polymer waveguide grating coupler with directionality enhancement, Opt. Commun. 463 (2020) 125418. https://doi.org/https://doi.org/10.1016/j.optcom.2020.125418.

[6] J. Zhang, C. Zhang, G. Liang, Y. Yang, Simulation research of highefficiency unidirectional vertical coupling grating couplers for optical through-silicon vias in 3D optoelectronic integrated circuits, Opt. Commun. 479 (2021) 126408. https://doi.org/https://doi.org/10.1016/j.optcom.2020.126408.

[7] X. Chen, H.K. Tsang, Polarization-independent grating couplers for siliconon-insulator nanophotonic waveguides, Opt. Lett. 36 (2011) 796-798. https://doi.org/10.1364/OL.36.000796. 
[8] C. Alonso-Ramos, L. Zavargo-Peche, A. Ortega-Moñux, R. Halir, I. MolinaFernández, P. Cheben, Polarization-independent grating coupler for micrometric silicon rib waveguides, Opt. Lett. 37 (2012) 3663-3665. https://doi.org/10.1364/OL.37.003663.

[9] Z. Xiao, T.-Y. Liow, J. Zhang, S.T.H. Silalahi, P. Shum, F. Luan, Mode Control in Planar Waveguide Grating Couplers With Double Surface Corrugation, IEEE Photonics Technol. Lett. 24 (2012) 1722-1725. https://doi.org/10.1109/LPT.2012.2213300.

[10] J.H. Song, F.E. Doany, A.K. Medhin, N. Dupuis, B.G. Lee, F.R. Libsch, Polarization-independent nonuniform grating couplers on silicon-oninsulator, Opt. Lett. 40 (2015) 3941-3944. https://doi.org/10.1364/OL.40.003941.

[11] A. Mudhafer, Z.S. Khaleel, R. Malallah, Excitation enhancement of surface plasmon polaritons from an annular plasmonic coupler based on internal corrugations and a central nanowire, J. Comput. Electron. (2021). https://doi.org/10.1007/s10825-021-01727-2.

[12] J. Zhang, J. Yang, H. Lu, W. Wu, J. Huang, S. Chang, Subwavelength TE/TM grating coupler based on silicon-on-insulator, Infrared Phys. Technol. 71 (2015) 542-546. https://doi.org/https://doi.org/10.1016/j.infrared.2015.06.018.

[13] J. Zhang, J. Yang, H. Lu, W. Wu, J. Huang, S. Chang, Polarizationindependent grating coupler based on silicon-on-insulator, Chin. Opt. Lett. 13 (2015) 91301. http://col.osa.org/abstract.cfm?URI=col-13-9-091301.

[14] N. Purwaha, A. Atieh, W.N. Ye, Broadband and polarization flexible SOI grating coupler based on sub-wavelength gratings with low back reflections, OSA Contin. 2 (2019) 1350-1357. https://doi.org/10.1364/OSAC.2.001350.

[15] T. Hao, A. Sánchez-Postigo, P. Cheben, A. Ortega-Moñux, W.N. Ye, DualBand Polarization-Independent Subwavelength Grating Coupler for Wavelength Demultiplexing, IEEE Photonics Technol. Lett. 32 (2020) 11631166. https://doi.org/10.1109/LPT.2020.3014640.

[16] H. Siampour, S. Kumar, S.I. Bozhevolnyi, Nanofabrication of Plasmonic Circuits Containing Single Photon Sources, ACS Photonics. (2017). https://doi.org/10.1021/acsphotonics.7b00374.

[17] K. Hajim, S. Tawfiq, A. Meki, Quantum Cryptography and a Quantum Key Distribution Protocol, Iraqi J. Laser. 3 (2004) 1-10. 
https://ijl.uobaghdad.edu.iq/index.php/IJL/article/view/151.

[18] P. Sibson, J.E. Kennard, S. Stanisic, C. Erven, J.L. O’Brien, M.G. Thompson, Integrated silicon photonics for high-speed quantum key distribution, Optica. 4 (2017) 172-177. https://doi.org/10.1364/OPTICA.4.000172.

[19] N. Bonod, J. Neauport, Diffraction gratings: from principles to applications in high-intensity lasers, Adv. Opt. Photon. 8 (2016) 156-199. https://doi.org/10.1364/AOP.8.000156.

[20] R. Larrea, A.M. Gutierrez, P. Sanchis, Design method for high performance grating couplers in photonic integrated circuits, Opt. Quantum Electron. 50 (2018) 341. https://doi.org/10.1007/s11082-018-1596-8.

[21] L.O. Skrebergene, Optimization and fabrication of a fiber-to-waveguide silicon-on-insulator grating coupler, NTNU, 2020.

[22] Thorlabs, PM630-HP, Https://Www.Thorlabs.Com/Drawings/8a6d8952d7d91b79-E8731100-CF3147F1-12A5821A0953BD9F/PM630-HP-SpecSheet.Pdf. (n.d.). https://www.thorlabs.com/thorproduct.cfm?partnumber=PM630-HP (accessed April 23, 2021).

[23] F.A. Khaleel, S.K. Tawfeeq, The spontaneous emission performance of a quantum emitter coupled to a hybrid plasmonic waveguide with specified output polarization for on-chip plasmonic single-photon source, Photonics Nanostructures - Fundam. Appl. 45 (2021) 100925. https://doi.org/https://doi.org/10.1016/j.photonics.2021.100925. 